\title{
Fabrication of Polymer-Based Micro Devices: Formulation and Study of the Paste
}

\author{
Nathalie Serra, Thomas Maeder, Olivier Gentsch, Peter Ryser \\ Laboratoire de Production Microtechnique, Ecole Polytechnique Fédérale de Lausanne, Station 17, 1015 Lausanne, Switzerland \\ http://lpm.epfl.ch; nathalie.serra@epfl.ch; thomas.maeder@epfl.ch \\ Original version: Procedia Engineering 5 (Proc. Eurosensors XXIV, September 5-8, 2010, Linz, Austria), 874-877, 2010 \\ (C) 2010 Published by Elsevier Ltd; http://hdl.handle.net/10.1016/j.proeng.2010.09.248
}

\begin{abstract}
We investigate in the present work the fabrication of polymer-based micro-fluidic and micro-mechanical devices using sacrificial, resistive/conductive pastes, through a screen-printing process. An organic sacrificial paste is first screen-printed onto a substrate, the future opening in the structure existing where the paste lies (channels, cavities...). Then, a resistive paste based on thermosetting polymer resin and graphite is deposited onto the previous layer. Finally, the sacrificial paste is removed at $150^{\circ} \mathrm{C}$ by sublimation through the composite, leaving therefore the desired pattern. For such applications, the key is naturally the formulation of the pastes. On a previous work, we already proposed a potential formulation for the organic sacrificial paste, based on polyols [1]. In the present paper, we will focus on the formulation of the cover paste, which must be adapted rheologically for the screen-printing process, but also chemically due to strong potential interactions between the sacrificial layer and the over-layer. Finally, micro-devices such as simple fluidic channels and suspended structures were produced and shown to be operational, demonstrating the high potential of our process.
\end{abstract}

Keywords:organic sacrificial layers; silicone resins; screen-printing; thick films

\section{Introduction}

In the micro-electromechanical systems (MEMS) field, sacrificial layers are widely used for fabrication of free-standing structures by surface or volume micromachining. However, many applications, due to size or cost reasons, do not require clean-room processes, and/or are just as well or better fabricated using other substrates than silicon. For instance, the thick-film and low-temperature co-fired ceramic (LTCC) technologies, principally based on glass/ceramic materials, are commonly used for sensors, and have recently seen considerable developments in structuration techniques [2-3] for fabrication of elements such as cantilevers, bridges, membranes and fluidic structures.

Nowadays, a move towards polymers is observed as their properties such as low cost, transparency and good biocompatibility make them particularly suitable for disposable biomedical applications. Micro-structures based on organic sacrificial layers, such as the combination of SU-8 (over-layer) and polypropylene carbonate (sacrificial material) have already been reported in the literature [4]. However the polypropylene carbonate decomposes around $300^{\circ} \mathrm{C}$ which remain too high for most polymer applications. Therefore we introduced [1] materials with significantly lower sacrificial layer removal temperatures, thereby considerably extending the application field of this process. Moreover, the materials employed are also chosen for their sublimation ability. This particular property has the advantage to allow closed structures such as micro-channels, cavities and membranes as the compounds sublime cleanly through the over-layer, without collapse due to surface tension, and do not require any etching process. Our organic sacrificial pastes are based on mixes of non-polymeric polyol-type organic materials (so-called plastic crystals). Polyols are widely used in industry for paints and coatings formulation, due to the viscosity and fast drying properties they give to the final paste. Some polyols have also attracted considerable attention due to their solid-solid order-disorder phase transitions, which make them interesting for thermal energy storage. In a previous work, we had characterized epoxygraphite resistive pastes for micro-heater manufacturing [5]. The point here is to analyze to what extent what we developed can be adapted to this other application. Particular care must therefore be given to the formulation of the over-layer. Various epoxy resins and silicones as thermosetting polymers were used in this study. A key factor in screen-printing process is the rheology of the paste (ca. honey-like consistency). In order to tune this property, the use of additives and/or solvents can be envisaged. We already tested different solvents that are well-suited for epoxies [5]. Here, this study was also extended to silicone, with the purpose of finding suitable solvents, i.e. having good solvency towards silicone resins, yet without chemical reactions. 


\section{Experimental}

Fabrication of the micro-fluidic and micro mechanical devices is performed by screen-printing: the sacrificial material is first screen-printed onto a substrate (here alumina) and dried around $80^{\circ} \mathrm{C}$. Then a second layer is over-printed and dried at $100^{\circ} \mathrm{C}$. Finally, the structures are placed at typically $150^{\circ} \mathrm{C}$ and sublimation of the organic paste occurs, leaving clean structures with the desired pattern (see Fig. 1).

Formulation of a structural over-layer that is both compatible with the sacrificial layers and screen-printable is here the key for successful fabrication of our structures. The organic sacrificial pastes we use were developed previously and are based on mixes of polyols partly dispersed and dissolved in cyclohexanol [1]. These were found to be well compatible (i.e. not significantly interact) with silicones and ethylcellulose, but not with epoxies, which are reactive to the polyol $\mathrm{OH}$ group, resulting in the destruction of the structure. In this paper, we focus on the formulation of silicone resin, filled with graphite to impart electrical conductivity and improve mechanical stability. Regular silicone resin (Q5-8401, Dow Corning, USA) was used as a matrix and graphite (KS4, ellipsoidal shape with $95 \%$ of the particles having a large axis smaller than $4 \mu \mathrm{m}$, Timcal, Switzerland) as a filler. Graphite loadings result in an increase of the final viscosity, which may prevent a good deposition by screen-printing. Therefore, the introduction of suitable high-boiling solvents, whose properties are given in Table 1, was studied to allow convenient tuning of the paste rheology.

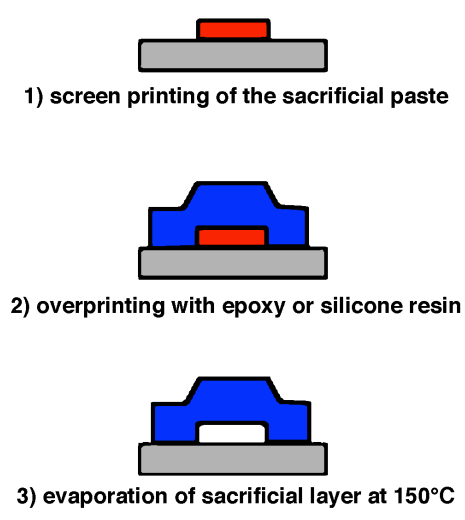

(a)

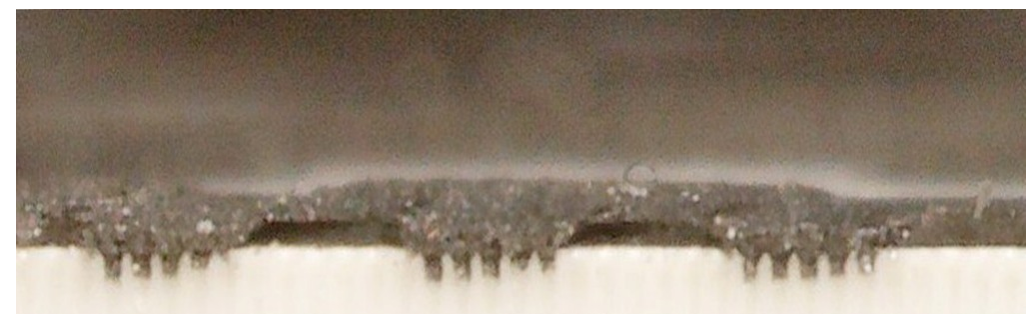

$1 \mathrm{~mm}$ alumina substrate

Fig.1: (a) Process of structures using sacrificial pastes; (b) Cross-section of a micro-channel after sublimation of the sacrificial paste

Table 1. Properties of tested solvents. $T b=$ boiling point; $M=$ molar mass; $d=$ density.

\begin{tabular}{llcccc}
\hline Name & CAS- $^{\circ}$ & $T_{b}\left({ }^{\circ} \mathrm{C}\right)$ & $M(\mathrm{~g} / \mathrm{mol})$ & $d\left(\mathrm{~kg} / \mathrm{m}^{3}\right)$ & Symbol \\
\hline Dodecane & $112-40-3$ & 215 & 170.33 & 750 & $\mathrm{~A} / \mathrm{B} 1$ \\
Octanol & $123-96-6$ & 196 & 130.23 & 827 & $\mathrm{~A} / \mathrm{B} 2$ \\
$(\mathrm{R})-(+)$ limonene & $5989-27-5$ & 175 & 136.24 & 840 & $\mathrm{~A} / \mathrm{B} 3$ \\
Tetraglyme & $143-24-8$ & 275 & 222.28 & 1009 & $\mathrm{~A} / \mathrm{B} 4$ \\
\hline
\end{tabular}

Solvents must be carefully chosen, they should be miscible with the resin, yet remain inert without chemical reaction. One would notice that this list includes also polar solvents (octanol and tetraglyme), which are not a priori suitable solvents for silicones. However, our sacrificial material having polar groups, it might be interesting to have also a certain percentage of a polar solvent in the over-layer, hence providing a desired wetting and adhesion between the layers. Therefore, knowing the behavior of these solvents with silicones would be of great use. In order to prove their efficiency and validate their use for paste formulation, mass loss tests were performed for $10 \%$ and $20 \%$ in volume of graphite. A second "qualitative" test consisting in rheological measurements was done at $10 \%$ vol. of graphite in order to classify the "solvative" nature of the materials. Finally, fabricated structures were tested and compared. 


\section{Results}

\subsection{Study of solvents}

A first series of mass loss experiments was performed in order to determine whether solvents evaporate completely during the curing process (see Fig.2a). A mass loss profile was made for $10 \%$ and $20 \%$ vol. graphite by measuring the composite mass after 30,60 and $120 \mathrm{~min}$ at $150^{\circ} \mathrm{C}$. Only the profile for $10 \%$ is shown here, as no significant difference between both loadings was observed. A/B0 samples correspond to the reference measurement, i.e. without solvent. Several conclusions can be drawn from these graphs. First, in each case, the reference sample shows a loss, probably corresponding to water. We can also notice that, whatever the solvent or the graphite loading, essentially all the loss occurs in the first thirty minutes, which is noticeably faster than with epoxies [1] and agrees with the higher permeability of silicones. The somewhat slower removal of tetraglyme is also in line with its high boiling point.

A second series of experiments consisted in rheological measurements. The dynamic viscosity was measured with a rotative viscosimeter, Rheomat RM180 from Mettler. No measurement was possible for the reference sample, the viscosity being too high for our device. Fig. $2 b$ reports the data for the four solvents.

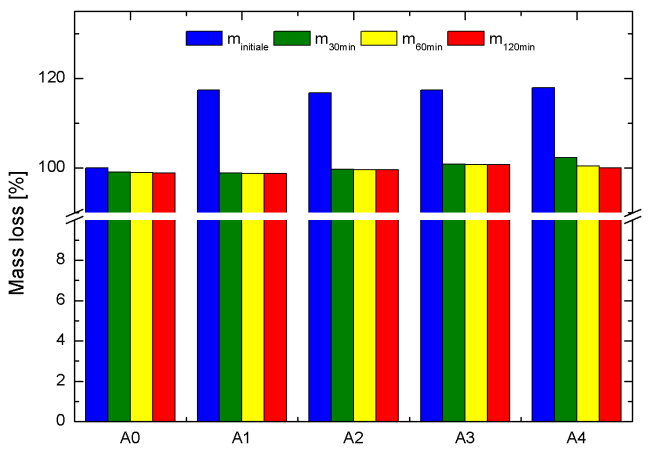

(a)

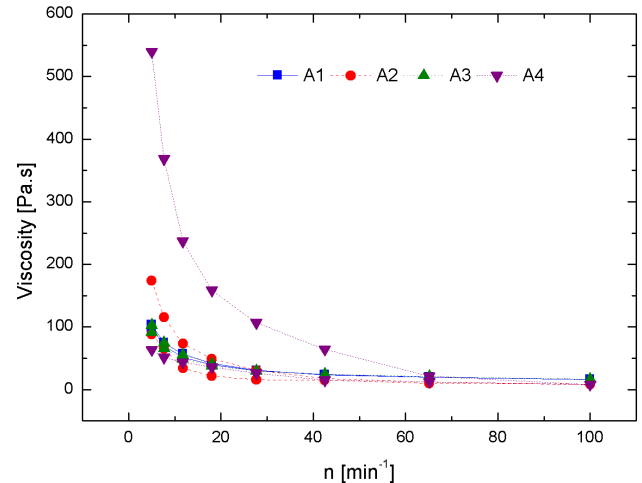

(b)

Fig 2. Relative composite mass vs. solvent in silicone with $10 \%$ vol KS4 (a); Viscosity measurements for the different solvents (b)

We can see that theoretical suppositions are confirmed, i.e. dodecane and limonene have a higher solvative power compared to the other two. However, it is also to notice that despite their chemical nature, the octanol and the tetraglyme also dilute after a certain time the resin. They form firstly a gel before being incorporated in the resin. This can be explained by the length of their "alcane" chain and their low polarity, which allow them to finally mix with the silicone. One will also notice that for these reasons, octanol will be incorporated faster in the resin than tetraglyme. Therefore, it would be possible to use them in combination with either dodecane or limonene.

\subsection{Fabrication of micro-channels}

Micro-channels using silicone as over-layer were fabricated, with the aim of studying the influence of a polar solvent on the good functionality of the structures. Silicone:graphite pastes were prepared using first only dodecane as solvent, than mixes of dodecane:octanol $(2: 1$ and 1:1). Test structures were dipped in water in order to check for leaks, air $(\approx$ 6 bar) was blown through the channel, and the maximum flow was determined in each case. The results (Table 2) show that without octanol, the structures tend to tear away from the substrate. On the other hand, an equal mix of dodecane and octanol gives the best results in terms of reproducible channel geometry.

Table 2: Influence of solvent formulation on the maximum air flow

\begin{tabular}{cc}
\hline Dodecane:octanol ratio & Max. Flow $[1 \mathrm{~N} / \mathrm{min}]$ \\
\hline $1: 0$ & (unreliable) \\
$2: 1$ & 1.7 \\
$1: 1$ & 2.8 \\
\hline
\end{tabular}




\section{Conclusion}

Screen-printable silicone:graphite structural over-layers compatible with an organic polyol sacrificial paste were successfully formulated. A study on solvents showed that dodecane and limonene have a high dilutive power on silicone resins, allowing therefore the tuning of paste rheology and extending the achievable range of filler loadings. We also demonstrated that adding a solvent with a polar group (i.e. octanol) to the formulation improved wetting and adhesion between the over-layer and the sacrificial material, thereby allowing reliable and convenient fabrication of printed micro-fluidic devices.

\section{Acknowledgements}

The authors would like to thank Timcal for the graphite powders. This work was partly financed by the Swiss CTI promotion agency.

\section{References}

[1] Serra N, Maeder T, Jacq C, Fournier Y, Ryser P. Screen-printed polymer-based microfluidic and micromechanical devices based on evaporable compounds. EMPC 2009 European Microelectronics and Packaging Conference and Exhibition, Rimini, Italy, June 15-18, 2009

[2] Lucat C, Ginet P, Ménil F. New sacrificial layer based screen-printed process for free-standing thick-films applied to MEMS. Journal of Microelectronics and Electronic Packaging 2007;4:86-92.

[3] Birol H, Maeder T, Ryser P. Application of graphite-based sacrificial layers for fabrication of LTCC membranes and microchannels. Journal of Micromechanics and Microengineering 2007;17:50-60

[4] Metz S, Jiguet S, Bertsch A, Renaud P. Polyimide and SU-8 microfluidic devices manufactured by heat-depolymerizable sacrificial material technique. Lab on a Chip 2004, 4:114-120

[5] Serra N, Maeder T, Lemaire P, Ryser P. Formulation of composite resistive pastes for fabrication of micro-heaters. Senasors and Actuators A: Physical 2010, Article in Press 